\title{
On some normability conditions
}

\author{
Tosun Terzioğlu*1, Murat Yurdakul ${ }^{* * 2}$, and Vyacheslav Zahariuta ${ }^{* * * 1}$ \\ ${ }^{1}$ Sabancı University, 34956 Tuzla, Istanbul, Turkey \\ ${ }^{2}$ Department of Mathematics, Middle East Technical University, 06531 Ankara, Turkey
}

Received 14 April 2003, revised 27 January 2005, accepted 19 May 2005

Published online 6 October 2005
Key words Locally convex spaces, quasi-normability, asymptotic normability, Vogt interpolation classes, Gelfand-Shilov property
MSC (2000) Primary: 46A03, 46A63; Secondary: 46A11, 46A20, 46A45

\begin{abstract}
Various normability conditions of locally convex spaces (including Vogt interpolation classes $D N_{\varphi}$ and $\Omega_{\varphi}$ as well as quasi- and asymptotic normability) are investigated. In particular, it is shown that on the class of Schwartz spaces the property of asymptotic normability coincides with the property $G S$, which is a natural generalization of Gelfand-Shilov countable normability (cf. [9, 25], where the metrizable case was treated). It is observed also that there are certain natural duality relationships among some of normability conditions.
\end{abstract}

(c) 2005 WILEY-VCH Verlag GmbH \& Co. KGaA, Weinheim

\section{Introduction}

The class of quasi-normable locally convex spaces, introduced by Grothendieck [11], was studied intensively in the last two decades (see e.g., [3-7, 18, 27]). In particular, there are various concrete characterizations of quasi-normability for Köthe spaces and their generalizations. Meise and Vogt [18] found that the class of quasinormable Fréchet spaces coincides with the union of classes $\Omega_{\varphi}$ (see, [28, 29, 31]) with $\varphi$ running through the set $\Phi$ of all strictly increasing functions from $\mathbb{R}_{+}$to itself. The class of asymptotically normable Fréchet spaces was introduced by Terzioğlu and Vogt in $[24,25]$ as a natural counterpart to the class of quasi-normable spaces; they showed also that asymptotically normable Fréchet spaces are those which admit a $D N_{\varphi}$-condition with $\varphi \in \Phi$.

Natural dual relationships among above and some related invariant classes of locally convex spaces are investigated here. Some of the previous results about normability conditions are extended to non-metrizable locally convex spaces. One of them (Theorem 2.14) tells that a Schwartz space is asymptotically normable if and only if it satisfies the condition $G S$, which is a natural generalization of Gelfand-Shilov's countable normability. The metrizable case was treated by in $[9,25]$; we simplify the proof, in particular, we avoid the closed graph theorem considerations (cf. [9, proof of Proposition 5, iii) $\Rightarrow$ i)]).

Finally, a concrete characterization of quasi-normability is considered for locally convex spaces with unconditional bases in terms of the condition $(G)$ (see, [4, Proposition 3.9]; cf. [7]).

\section{Interpolational and normability properties}

We use the standard terminology of theory of locally convex spaces as in $[15,17]$. On a locally convex space $E$ we always consider a basic system of seminorms $\left\{|\cdot|_{p}, p \in P\right\}$, which means that the corresponding system of unit balls

$$
U_{p}:=\left\{x \in E:|x|_{p} \leq 1\right\}, \quad p \in P,
$$

* Corresponding author: e-mail: tosun @ sabanciuniv.edu, Phone: 902164835010 , Fax: 902164839013

** e-mail: myur@metu.edu.tr, Phone: 903122105389 , Fax: 903122101282

*** e-mail: zaha@ sabanciuniv.edu, Phone: 902164839033 , Fax: 902164839550 
forms a base of neighborhoods of $0 \in E$. The set $P$ is a directed set with the natural partial order

$$
p \leq q \Longleftrightarrow|x|_{p} \leq|x|_{q}, x \in E .
$$

On the strong dual $E^{\prime}$ we have the system of polar norms

$$
\left|x^{\prime}\right|_{p}^{*}:=\sup \left\{\left|x^{\prime}(x)\right|: x \in E,|x|_{p} \leq 1\right\}, \quad x^{\prime} \in E^{\prime}, \quad p \in P .
$$

For $p \in P$ the Banach space $E_{p}$ is a completion of the space $E / k e r|\cdot|_{p}$, considered with the corresponding quotient norm, and $I_{q, p}: E_{q} \rightarrow E_{p}$ is the natural linking map if $q \geq p$.

We denote by $\mathcal{B}(E)$ the locally convex bornology of $E$ consisting of all absolutely convex bounded sets in $E$. For each $M \in \mathcal{B}(E)$ we consider its gauge norm $|x|_{M}$ and its polar norm $\left|x^{\prime}\right|_{M}^{*}:=\sup \left\{\left|x^{\prime}(x)\right|: x \in M\right\}$ on $E^{\prime}$.

By $\lambda(A)$ we denote the Köthe space defined by the matrix $A=\left(a_{i, p}\right)$ with $0 \leq a_{i, p} \leq a_{i, p+1}$ for all $(i, p) \in \mathbb{N}^{2}$.

Now we discuss the direct generalization of two important classes, introduced by Vogt for Fréchet spaces ([28, 29, 31]). Suppose $\varphi: \mathbb{R}_{+} \rightarrow \mathbb{R}_{+}$is a strictly increasing function.

Definition 2.1 A locally convex space $E$ satisfies the property $D N_{\varphi}\left(E \in\left(D N_{\varphi}\right)\right)$ if there is $p \in P$ and a map $\rho \in P^{P}$ such that for each $q \in P$ there is $C=C(q)>0$ such that

$$
|x|_{q} \leq C \varphi(t)|x|_{p}+\frac{1}{t}|x|_{\rho(q)}, \quad x \in E, \quad t>0 .
$$

Definition 2.2 A locally convex space $E$ satisfies the property $\Omega_{\varphi}\left(E \in\left(\Omega_{\varphi}\right)\right)$ if there is a map $\sigma \in P^{P}$ such that for any $p \in P$ and any $r \in P$ there is $C=C(p, r)>0$ such that

$$
\left|x^{\prime}\right|_{\sigma(p)}^{*} \leq C \varphi(t)\left|x^{\prime}\right|_{r}^{*}+\frac{1}{t}\left|x^{\prime}\right|_{p}^{*}, \quad x^{\prime} \in E^{\prime}, \quad t>0 .
$$

This condition can be written equivalently in terms of neighborhoods as

$$
U_{\sigma(p)} \subset C \varphi(t) U_{r}+\frac{1}{t} U_{p}, \quad t>0 .
$$

We say that $E$ satisfies the property $\widehat{\Omega}_{\varphi}$ (and write $E \in\left(\widehat{\Omega}_{\varphi}\right)$ ) if the constant in Eq. (2.2) can be taken in the form $C=B(p) \cdot D(r)$.

The classes $\left(D N_{\varphi}\right)$ and $\left(\Omega_{\varphi}\right)$ have been proved to be of a great importance in the study of linear topological structure of locally convex spaces (see, e.g., $[10,14,17,18,24-26,28,29,31]$ ). Both properties, $D N_{\varphi}$ and $\Omega_{\varphi}$, are linear topological invariants; the first is inherited by subspaces, while the latter by quotient spaces ([18], see also [31]).

Remark 2.3 (Cf. [28, 10]) By iterating Definitions 2.1, 2.2 one can easily derive $\Omega_{\varphi}=\Omega_{\psi}, D N_{\varphi}=$ $D N_{\psi}$ if $\psi(t)=\varphi\left(t^{\alpha}\right)$ for any $\alpha>0$.

Definition 2.4 A locally convex space $E$ satisfies the strict $\Omega_{\varphi}$ property $\left(E \in\left(s \Omega_{\varphi}\right)\right)$ if there exist a map $\sigma \in P^{P}$ and a set $M \in \mathcal{B}(E)$ such that for any $p \in P$ there is a constant $C=C(p)>0$ such that

$$
U_{\sigma(p)} \subset C \varphi(t) M+\frac{1}{t} U_{p}, \quad t>0,
$$

(due to the assumption that $U_{p}$ is a base of neighborhoods of zero in $E$, we can even assume, without loss of generality, that $C(p)=1)$.

By Grothendieck ([11], p. 107), a locally convex space $E$ is said to be quasi-normable if there is a map $\sigma \in P^{P}$ such that for each $p \in P$ and $\varepsilon>0$ there exists $M \in \mathcal{B}(E)$ such that $U_{\sigma(p)} \subset M+\varepsilon U_{p}$. We say that $E$ is strictly quasi-normable if there is $M \in \mathcal{B}(E)$ and a map $\sigma \in P^{P}$ such that for each $p \in P$ and $\delta>0$ there exists $\Delta>0$ such that

$$
U_{\sigma(p)} \subset \Delta M+\delta U_{p} .
$$


We say that $E$ is perfectly quasi-normable $(E \in(p Q N))$ if the constant $\Delta$ in Eq. (2.5) can be taken in the form $\Delta=C(p) D(\delta)$. It is well-known that for Fréchet spaces all these properties coincide (see [11, 13]). In general, all inclusions $(Q N) \subset(s Q N) \subset(p Q N)$ are proper; in particular, the space $\omega^{*}$ is quasi-normable, but fails to be strictly quasi-normable. For the dual space $E^{\prime}$, besides strict quasi-normability, we consider the stronger property $\overline{s Q N}$, demanding in Eq. (2.5) the equicontinuity of $M$ (if $E$ is quasi-barrelled these properties of $E^{\prime}$ coincide). Meise and Vogt [18] proved that a Fréchet space $E$ is quasi-normable if and only if it belongs to the class $\left(\Omega_{\varphi}\right)$ for some $\varphi$.

Theorem 2.5 Suppose a metrizable locally convex space E satisfies both properties $\Omega_{\varphi}$ and $Q N$. Then $E \in\left(s \Omega_{\psi}\right)$ if $\psi(t) / \varphi(t) \rightarrow \infty$ as $t \rightarrow \infty$.

Proof. Since $E$ is metrizable, we may take $P=\mathbb{N}$. From $E \in(Q N)$ we derive

$$
U_{\pi(r)} \subset M(r, \varepsilon)+\varepsilon U_{r}, \quad \varepsilon>0, \quad r \in \mathbb{N},
$$

with some $\pi \in \mathbb{N}^{\mathbb{N}}$ and $M(r, \varepsilon) \in \mathcal{B}(E)$.

On the other hand, from $E \in\left(\Omega_{\varphi}\right)$ we derive, without loss of generality, that the relation Eq. (2.3) holds with the constant depending only on $r$. Otherwise, since in the metrizable case the conditions $\Omega_{\varphi}$ and $\widehat{\Omega}_{\varphi}$ are obviously equivalent, the constant $C$ can be chosen in the form $C=B(p) \cdot D(r), B(p) \geq 1$, and we obtain this property by changing $\sigma \in \mathbb{N}^{\mathbb{N}}$ to $\widetilde{\sigma} \in \mathbb{N}^{\mathbb{N}}$ so that $B(p) U_{\widetilde{\sigma}(p)} \subset U_{\sigma(p)}, p \in \mathbb{N}$. Thus, taking in this relation $\pi(r)$ instead $r$, we get

$$
U_{\sigma(p)} \subset C(\pi(r)) \varphi(t) U_{\pi(r)}+\frac{1}{t} U_{p}, \quad t>0, \quad p, r \in \mathbb{N} .
$$

Now we choose $\tau_{r} \uparrow \infty$ so that $\varphi(t) C(\pi(r)) \leq \psi(t)$ for $t \geq \tau_{r}$, and define

$$
\varepsilon_{r}:=\left(C(\pi(r)) \varphi\left(\tau_{r+1}\right) \tau_{r+1}\right)^{-1}, \quad M_{r}:=\left(M\left(r, \varepsilon_{r}\right) \cap 2 U_{r}\right), \quad r \in \mathbb{N} .
$$

Puting the estimate (2.6) considered with $\varepsilon=\varepsilon_{r}$ into Eq. (2.7), we obtain, due to our choice of $\tau_{r}$ and $\varepsilon_{r}$,

$$
U_{\sigma(p)} \subset \psi(t) M_{r}+\frac{2}{t} U_{p}, \quad \tau_{r} \leq t \leq \tau_{r+1}, \quad r \geq p, \quad p \in \mathbb{N} .
$$

Now we construct the set $M$ as a convex hull of the union of the sets $M_{r}, r \in \mathbb{N}$. It is easy to see that $M \in \mathcal{B}(E)$. From Eq. (2.8) we obtain

$$
\frac{1}{2} U_{\sigma(p)} \subset \psi(t) M+\frac{1}{t} U_{p}, \quad t \geq \tau_{p}, \quad p \in \mathbb{N} .
$$

Finally, choosing $\rho \in \mathbb{N}^{\mathbb{N}}$ so that $U_{\rho(p)} \subset\left(\frac{1}{2} U_{\sigma(p)}\right) \cap\left(\frac{1}{\tau_{p}} U_{p}\right)$, we get

$$
U_{\rho(p)} \subset \psi(t) M+\frac{1}{t} U_{p}, \quad t>0, \quad p \in \mathbb{N},
$$

that is $E \in\left(s \Omega_{\psi}\right)$.

Remark 2.6 Without assumption on quasinormability the statement may fail. Namely, Bonet and Dierolf [8] gave an example of a non-quasinormable metrizable (non-complete) locally convex space $F$, which is a dense linear subspace of a quasi-normable Fréchet space $E$. Since, by [18], $E$ belongs to some class $\left(\Omega_{\varphi}\right)$, its dense linear subspace $F$ also belongs to this class. But $F$ cannot satisfy any $s \Omega_{\varphi}$, since otherwise it would be quasinormable.

Corollary 2.7 Let a Fréchet space E have the property $\Omega_{\varphi}$. Then E belongs to the class $\left(s \Omega_{\psi}\right)$, if $\psi(t) / \varphi(t) \rightarrow$ $\infty$ as $t \rightarrow \infty$.

Proof. Since in this case, by [18], $E \in(Q N)$ is a consequence of $E \in\left(\Omega_{\varphi}\right)$, we can apply Theorem 2.5.

Under an additional restriction we can prove this fact with $\psi=\varphi$. 
Proposition 2.8 Suppose that a function $\varphi$ is such that

$$
\lim _{t \rightarrow \infty} \varphi\left(t^{\alpha}\right) / \varphi(t)=0
$$

for some $\alpha>0$. Then a Fréchet space E satisfies $\Omega_{\varphi}$ if and only if $E \in\left(s \Omega_{\varphi}\right)$.

In the proof of Theorem 2.5 we have basically followed Vogt ([28, Lemma 1.4] and Meise-Vogt [17, Lemma 29.16]), where the case $\varphi(t)=t$, very important for applications, is considered (the property $\Omega_{\varphi}$ in that case is called also $D_{2}$ or $\bar{\Omega}$ ). Those considerations relate to so-called "dead-end spaces", which are useful in studying of bases in certain spaces, especially in finite centers of Hilbert scales and in some spaces of analytic functions (see, e.g., $[1,2,19-21,32,33,36]$ ).

A locally convex space $E$ is said to be asymptotically normable (cf. [25]) (we write $E \in(A N)$ ) if there is $p \in P$ and a map $\rho \in P^{P}$ such that for each $q \in P$ the $q$-topology coincides with the $p$-topology on the unit ball $U_{\rho(q)}$, which means that for each $\delta>0$ there is $\Delta=\Delta(q, \delta)>0$ such that

$$
|x|_{q} \leq \Delta|x|_{p}+\delta|x|_{\rho(q)}, \quad x \in E
$$

We say that $E$ is perfectly asymptotically normable $(E \in(p A N))$ if the constant $\Delta$ in Eq. (2.11) can be taken in the form $C(q) \cdot D(\delta)$. For non-metrizable locally convex spaces these two properties may not coincide.

Terzioğlu and Vogt [24] introduced also the following property, which is a weakening of $A N$ : a locally convex space $E$ is locally normable $(E \in(L N))$ if there is $p \in P$ such that on each $M \in \mathcal{B}(E)$ the $p$-topology coincides with the topology of $E$.

We say that a locally convex space $E$ is strictly locally normable $(E \in(s L N))$ if there is $p \in P$ such that for each $M \in \mathcal{B}(E)$ there is $L \in \mathcal{B}(E)$ such that the $p$-topology coincides with the $L$-topology on $M$, or

$$
|x|_{L} \leq \Delta|x|_{p}+\delta|x|_{M}, \quad x \in E
$$

for arbitrary $\delta>0$ and $\Delta=\Delta(M, \delta)>0$.

Proposition 2.9 A locally convex space $E$ is perfectly asymptotically normable if and only if $E \in\left(D N_{\varphi}\right)$ for some function $\varphi$.

This is an easy extension of Terzioğlu-Vogt's result [25] for Fréchet spaces.

The property $s L N$ is intermediate between $p A N$ and $L N$ : the inclusion $(s L N) \subset(L N)$ is obvious, while $(p A N) \subset(s L N)$ will be proved in the next section (Lemma 3.6). For Montel spaces the relations among some of above properties are more transparent.

Theorem 2.10 Let $E$ be a Montel locally convex space. Then

(a) $E \in(L N)$ if and only if it admits a continuous norm;

(b) $E \in(s L N)$ if and only if E admits a continuous norm and is co-Schwartz;

(c) $E$ is quasi-normable if and only if it is Schwartz;

(d) $E \in(s Q N)$ if and only if it is Schwartz and admits a total bounded set.

Pro of. Notice first that (c) is due to Grothendieck (see, e.g., [13, Section 10.7]).

(a) Only the if-part needs a proof. Since $E$ is Montel, any $M \in \mathcal{B}(E)$ considered with the topology $\tau(E)$ induced from $E$ is a compact topological space. Suppose that there is $p \in P$ such that $|\cdot|_{p}$ is a norm. Then the $p$-topology is a Hausdorff topology on $M$ coarser than $\tau(E)$, so these topologies must coincide.

(b) Let $E \in(s L N)$. Since $E$ is Montel, any $M \in \mathcal{B}(E)$ is precompact in $E$, but by the definition of $s L N$ there are $p \in P$ and $L \in \mathcal{B}(E)$ such that the topology $\mathcal{T}_{L}$, as considered on $M$, coincides with the topology defined by $|\cdot|_{p}$. Hence $M$ is also precompact with respect to $L$, which means that $E$ is co-Schwartz. Suppose now that $E$ is co-Schwartz. Then, putting the norm $|\cdot|_{L}$ instead of $|\cdot|_{q}$ everywhere in the proof of (a), we obtain, by the same token, that $E \in(s L N)$.

(d) Let $E \in(s Q N)$. Then, due to (c), $E$ is Schwartz, and it is easy to see that the set $M$ from Eq. (2.5) is total. Suppose that $E$ is Schwartz and admits a total set $M \in \mathcal{B}(E)$. Then for any $p \in P$ there is $q \in P$ such that for each $\delta$ there is a finite set $T \subset U_{q}$ such that

$$
U_{q} \subset T+\delta / 2 U_{p}
$$


Applying the totality of $M$ we get

$$
T \subset \bigcup_{n=1}^{\infty} n M+\delta / 2 U_{p} .
$$

Since $T$ is finite, we can find $\Delta \in \mathbb{N}$ so that $T \subset \Delta M+\delta / 2 U_{p}$. Hence the relation (2.5) holds, and $E \in(s Q N)$.

The following notion is a natural extension of the concept of countable normability (Gelfand-Shilov, see, e.g., [9]).

Definition 2.11 We say that a locally convex space $E$ satisfies the Gelfand-Shilov property $(E \in(G S))$ if there is a basic system of norms $\left\{\|\cdot\|_{k}, k \in K\right\}$ defining the topology of $E$ and such that for each $k \in K$ and every $m \in K$ provided $m \geq k$ the linking map $I_{m, k}: E_{m} \rightarrow E_{k}$ is injective, where $E_{k}$ stands for a completion of $\left(E,\|\cdot\|_{k}\right)$.

Let $A=(a(t, p))_{t \in T, p \in P}$, where $T$ is any set, $P$ is a directed set, $0 \leq a(t, p) \leq a(t, q)$ for $p \leq q$. Lez $T_{p}:=\{t \in T: a(t, p)>0\}, p \in P$, and $T=\bigcup_{p \in P} T_{p}$. We use a term weighted sup-space for the space

$$
\lambda_{\infty}(T, A):=\left\{u \in \mathbb{R}^{T}:|u|_{p}:=\sup \{|u(t)| a(t, p): t \in T\}<\infty, p \in P\right\}
$$

endowed with the locally convex topology defined by the system of seminorms $\left\{|\cdot|_{p}, p \in P\right\}$; if $a(t, p)$ is positive for all $t, p$, the space (2.13) is called a weighted sup-norm space (see, e.g., [30]). Denote by $l_{\infty}\left(a^{(p)}\right)$ the Banach space consisting of all functions $u \in \mathbb{R}^{T_{p}}$ with a finite sup-norm, defined by the weight $a^{(p)}:=a(t, p), t \in T_{p}$, and consider the map $R_{p}: \lambda_{\infty}(T, A) \rightarrow l_{\infty}\left(a^{(p)}\right)$ such that $R_{p}(u):=u \mid T_{p}$.

Definition 2.12 Let $F$ be a subspace of the weighted sup-space defined by Eq. (2.13) and $F_{p}$ be the closure of $R_{p}(F)$ in $l_{\infty}\left(a^{(p)}\right), p \in P$. Then we say that a subspace $F$ is well-imbedded into this space if, for any $p \leq q$, the conditions $u \in F_{q}$ and $u(t)=0, t \in T_{p}$, imply that $u(t) \equiv 0$ on $t \in T_{q}$.

The following lemma is a generalization of Vogt's result [30]; we are following basically his proof with some simplifications in the part (ii) $\Rightarrow$ (i).

Lemma 2.13 Let $E$ be a locally convex space and $\left\{|\cdot|_{p}, p \in P\right\}$ be any system of seminorms defining its topology. Then the following statements are equivalent:

(i) $E \in(G S)$;

(ii) There exists $p \in P$ and a mapping $\rho \in P^{P}$ such that for each $q \geq p$ any $|\cdot|_{\rho(q)}$-Cauchy sequence $\left\{x_{k}\right\} \subset E$ with $\left|x_{k}\right|_{p} \rightarrow 0$ converges to 0 also by $|\cdot|_{q}$;

(iii) $E$ is isomorphic to a well-imbedded subspace of some weighted sup-space of the space defined by Eq. (2.13).

Proof. (ii) $\Rightarrow$ (i). Taking $p$ and $\rho$ as in (ii), we define for any $q \geq p$ a new norm by the relation

$$
\|x\|_{q}:=\inf \left\{\lim _{k \rightarrow \infty}\left|x_{k}\right|_{\rho(q)}\right\}, \quad x \in E
$$

where the infimum is over the set of all $\rho(q)$-Cauchy sequences $\left(x_{k}\right)$ in $E$ such that $\left|x_{k}-x\right|_{q} \rightarrow 0$. It is obvious that this norm satisfies the estimates

$$
|x|_{q} \leq\|x\|_{q} \leq|x|_{\rho(q)}, \quad x \in E .
$$

Hence the system of norms $\left\{\|\cdot\|_{q}\right\}_{q \in Q}$ with $Q:=\{q \in P: p \leq q\}$ defines the original topology of $E$.

Denote by $\widetilde{E}_{q}$ the completion of the normed space $\left(E,\|\cdot\|_{q}\right), q \in Q$, and by $J_{r, q}: \widetilde{E}_{r} \rightarrow \widetilde{E}_{q}, r \geq q$, the corresponding unique extension of the identity. In particular, since $\rho(p)=p$ is available in Eq. (2.11), we can assume that $\|\cdot\|_{p}=|\cdot|_{p}$ and $\widetilde{E}_{p}=E_{p}$. By the definition (2.14), we may identify $\widetilde{E}_{q}$ with the quotient space $E_{\rho(q)} / N_{q}$, where $N_{q}=\operatorname{ker} I_{\rho(q), q}$. Let $\tau_{q}: E_{\rho(q)} \rightarrow E_{\rho(q)} / N_{q}$ be the canonical quotient map. 
The condition (ii) means that for any $q$ the linking map $I_{q, p}$ is injective as considered on the image $R_{q}:=I_{\rho(q), q}\left(E_{\rho(q)}\right), q \in P$. Therefore the map $\sigma_{q}: \widetilde{E}_{q}=E_{\rho(q)} / N_{q} \rightarrow R_{q}$, such that $I_{\rho(q), q}=\sigma_{q} \circ \tau_{q}$, is a bijection, which implies the injectivity of the map $J_{q, p}=I_{q, p} \circ \sigma_{q}$ for every $q \in Q$. Hence we deduce now the injectivity of the mapping $J_{r, q}, r \geq q$, from the obvious relation $J_{r, p}=J_{q, p} \circ J_{r, q}$. So, the new equivalent system of norms (2.14), $q \in Q$, on $E$ is constructed, which complies with the property $G S$ (Definition 2.11).

(i) $\Rightarrow$ (iii). Let $E \in(G S)$ and $\left\{\|\cdot\|_{k}, k \in K\right\}, \widetilde{E}_{k}, I_{m, k}$ be as in Definition 2.11; let $\|\cdot\|_{k}^{*}$ be the corresponding polar norms on the strong dual $E^{\prime}$. Set $T=E^{\prime} \backslash\{0\}, T_{k}=\left\{t \in T:|t|_{k}^{*}<\infty\right\}$, and $a(t, k):=1 /|t|_{k}^{*}$ if $t \in T_{k}$ and $a(t, k):=0$ if $t \in T \backslash T_{k}$.

Consider the operator $S: E \rightarrow \lambda_{\infty}(T, A)$ defined by $(S x)(t):=t(x), t \in T$. Since

$$
\|x\|_{k}=\sup \{|t(x)| a(t, k): t \in T\}, \quad x \in E, \quad k \in K,
$$

this operator is an isomorphism of $E$ onto its image $F$. Let $F_{k}$ be the spaces, derived from $F$ as in Definition 2.12 . Then, due to Eq. (2.16), the formula $S_{k}(x)(t)=t(x), t \in T_{k}$, defines an isometric surjection $S_{k}: \widetilde{E}_{k} \rightarrow F_{k}$ for any $k$. Hence the natural map $R_{m, k}: F_{m} \rightarrow F_{k}$, defined as a restriction from $T_{m}$ onto $T_{k}, k \leq m$, can be represented in the form $R_{m, k}=S_{m}^{-1} \circ I_{m, k} \circ S_{k}$. Since, by the assumption, $I_{m, k}$ is an injection, so is $R_{m, k}$, which means that the subspace $F$ is well-imbedded into $\lambda_{\infty}(T, A)$.

(iii) $\Rightarrow$ (ii). We obtain this inclusion, taking into account that, on the one hand, any well-imbedded subspace of a sup-space (2.13) satisfies obviously the condition (ii) with any $p$ and $\rho(q) \equiv q$ and, on the other hand, the condition (ii) is invariant under isomorphisms.

The next theorem generalizes Terzioğlu-Vogt's result $([24,25])$.

Theorem 2.14 For a locally convex space $E$ to be asymptotically normable it is necessary and, if $E$ is Schwartz, sufficient that $E \in(G S)$.

Proof. Suppose first that $E \in(A N)$ and $\left\{|\cdot|_{q}, q \in P\right\}$ is a basic system of seminorms in it. Then there is $p \in P$ and a non-decreasing map $\rho \in P^{P}$ such that the condition (2.11) holds. Hence, we get $E \in(G S)$ by Proposition 2.13, since the condition (ii) follows obviously from Eq. (2.11).

Suppose now that a Schwartz locally convex space $E$ satisfies the property $G S$, so that it can be endowed with a basic system of norms from Definition 2.11. Choose $\rho \in K^{K}$ such that $U_{\rho(q)}$ is precompact in the $q$-topology for all $q \in K$. It is sufficient to show that, given $p \in K$, for any $q \geq p$ the $q$-topology coincides with the $p$-topology on the ball $U_{\rho(q)}$, which will provide $E \in(A N)$. Indeed, supposing the contrary, we get a sequence $\left\{x_{j}\right\} \subset U_{\rho(q)}$ which converges to 0 in the $p$-topology but $\left|x_{j}\right|_{q} \geq \delta>0$ for all $j$. Then, using the precompactness of $U_{\rho(q)}$ in the $q$-topology, we find a subsequence $\left\{x_{j_{n}}\right\}$ which is $q$-Cauchy but does not converge to 0 in the $q$-topology. The sequence $\left\{x_{j_{n}}\right\}$ generates a non-zero element $z \in E_{q}$ such that $I_{q, p}(z)=0$, which is in contradiction with Definition 2.11 .

For a given locally convex space $E$ and a fixed $p \in P$ we consider a new system of seminorms defined by

$$
\|x\|_{q}^{(p)}:=\sup \left\{|t(x)|:|t|_{q}^{*} \leq 1,|t|_{p}^{*}<\infty\right\}, \quad q \in P, \quad q \geq p
$$

and denote by $\mathcal{T}_{E}^{(p)}$ the topology on $E$ generated by it (note that this topology is Hausdorff if and only if $|\cdot|_{p}$ is a norm). The following two notions, introduced in $[22,23,30]$, are defined here in a slightly different equivalent form.

Definition 2.15 $E$ is called

(a) locally closed $(E \in(L C))$ if the topology $\mathcal{T}_{E}^{(p)}$ coincides with the original topology of $E$ for some $p \in P$;

(b) locally admissible $(E \in(y))$ if the topology $\mathcal{T}_{E}^{(p)}$ is $\left(E, E^{\prime}\right)$-admissible for some $p \in P$.

Vogt proved in [30] that $E$ is locally closed if and only if it is isomorphic to a subspace of some weighted sup-norm space. The following characterization of locally admissible spaces is a simple corollary from this result.

Proposition 2.16 A locally convex space E is locally admissible if and only if it is weakly isomorphic to a subspace of some weighted sup-norm space (2.13).

Now we compare the properties $A N$ and $L C$. 
Proposition 2.17 If $E$ is asymptotically normable then it is locally closed.

Proof. Suppose $E \in(A N)$. Then there exist $p \in P$ and $\rho \in P^{P}$ such that the condition (2.11) holds for every $q \in P$ and each $\delta>0$ with some $\Delta=\Delta(q, \delta)$. Rewriting this condition in equivalent form in terms polars of neighborhoods, we obtain

$$
U_{q}^{\circ} \subset \Delta U_{p}^{\circ}+\delta U_{\rho(q)}^{\circ} \subset E_{p}^{\prime}+\delta U_{\rho(q)}^{\circ}, \quad \delta>0, \quad q \in P .
$$

Take an arbitrary $t \in U_{q}^{\circ}$. Applying Eq. (2.18), we find $t_{n} \in E_{p}^{\prime}$ such that

$$
\left|t(x)-t_{n}(x)\right| \leq 1 / n|x|_{\rho(q)}, \quad x \in E
$$

from where we derive also that $t_{n} \in(1+1 / n) U_{\rho(q)}^{\circ} \subset 2 U_{\rho(q)}^{\circ}$. From these considerations we conclude that

$$
U_{q}^{\circ} \subset \overline{E_{p}^{\prime} \cap 2 U_{\rho(q)}^{\circ}}, \quad q \in P,
$$

where the closure is considered in the weak topology $\sigma\left(E^{\prime}, E\right)$. Hence $|x|_{q} \leq 2\|x\|_{\rho(q)}^{(p)}, q \in P$, which means that the topology $\mathcal{T}_{E}^{(p)}$ coincides with the topology of $E$. So, $E \in(L C)$.

Using previous considerations and results from $[25,30]$ we get that, for Schwartz spaces, the above properties have especially simple description.

Theorem 2.18 Let E be a Schwartz space. Then the following are equivalent:

(1) $E$ is asymptotically normable;

(2) E has the Gelfand-Shilov property;

(3) E is locally closed;

(4) E is isomorphic to a subspace of some weighted sup-norm space.

If $E$ is also Fréchet space then this list may be extended by

(5) E is locally admissible;

(6) E is isomorphic to a subspace of $l_{\infty} \widehat{\otimes}_{\pi} \lambda(A)$, where $\lambda(A)$ is a nuclear Köthe space.

\section{Duality}

Here we study certain dual relationships between some of the properties we have already considered.

Theorem 3.1 If a locally convex space E satisfies $D N_{\varphi}$ then its strong dual $E^{\prime}$ satisfies $\widehat{\Omega}_{\varphi}$.

Proof. Suppose that $E \in\left(D N_{\varphi}\right)$, then Eq. (2.1) is true. Without loss of generality we assume that $r=$ $\rho(q) \geq q, q \in P$, hence the system of seminorms

$$
\left\{|x|_{r}, r \in R:=\rho(P)\right\}
$$

defines the original topology on $E$.

We will use the special base $\mathcal{M}$ of bornology $\mathcal{B}(E)$ consisting of sets

$$
M=M_{\alpha}:=\left\{x \in E:|x|_{M}:=\sup \left\{\alpha(r)|x|_{r}: r \in R\right\} \leq 1\right\}
$$

with $\alpha$ running over the set of all functions $\alpha: R \rightarrow(0,1]$. Given an arbitrary $M=M_{\alpha} \in \mathcal{M}$, we multiply the inequality Eq. (2.1) by $\frac{\alpha(\rho(q))}{C(q)}$ and take the supremum by $q$ over $P$. Then we get the estimate (we assume that $C(q) \geq 1)$

$$
\sup \left\{\frac{\alpha(\rho(q))}{C(q)}|x|_{q}: q \in P\right\} \leq \varphi(t)|x|_{p}+\frac{1}{t}|x|_{M}, \quad t>0 .
$$


It is clear that the set $A=\left\{x \in E:|x|_{q} \leq \frac{C(q)}{\alpha(\rho(q))}, q \in P\right\}$ is bounded in $E$ so there is $L=M_{\beta} \in \mathcal{M}$ such that $A \subset L$, hence Eq. (3.1) implies that

$$
|x|_{L} \leq \varphi(t)|x|_{p}+\frac{1}{t}|x|_{M}, \quad t>0
$$

Finally we have that for every $M \in \mathcal{M}$ there is $L \in \mathcal{M}$ and an equicontinuous (hence bounded) set $U_{p}^{\circ}$ in $E^{\prime}$ such that

$$
L^{\circ} \subset \varphi(t) U_{p}^{\circ}+\frac{1}{t} M^{\circ}, \quad t>0 .
$$

Since $\mathcal{M}^{\circ}:=\left\{M^{\circ}: M \in \mathcal{M}\right\}$ is a basis of neighborhoods of $E^{\prime}$, we have that the space $E^{\prime}$ satisfies the property $\widehat{\Omega}_{\varphi}$.

For a given Köthe space $E=\lambda(A)$ and sequence of positive numbers $\gamma=\left(\gamma_{i}\right)$ we introduce the notation

$$
\mathbf{B}(\gamma):=\left\{x=\left(\xi_{i}\right) \in \lambda(A): \sum_{i=1}^{\infty}\left|\xi_{i}\right| \gamma_{i} \leq 1\right\} .
$$

Theorem 3.2 Let a Montel Köthe space $E=\lambda(A)$ admit a continuous norm and satisfy $\Omega_{\varphi}$. If $\psi(t) / \varphi(t) \rightarrow \infty$ as $t \rightarrow \infty$, then the strong dual $E^{\prime}$ satisfies $D N_{\psi}$. If Eq. (2.10) holds for some $\alpha>0$, then $E^{\prime} \in\left(D N_{\varphi}\right)$.

We need the following quite well-known fact (see, e.g., [4]); its proof is represented here for the sake of completeness.

Lemma 3.3 Let $E=\lambda(A)$ be a Montel space admitting a continuous norm and $\Pi$ be a family of all nondecreasing positive integer-valued sequences $\left(p_{i}\right)$ tending to $\infty$. Then the collection

$$
C\left\{\mathbf{B}\left(\left(a_{i, p_{i}}\right)\right)\right\}, \quad\left(p_{i}\right) \in \Pi, \quad C>0,
$$

forms a base of bornology $\mathcal{B}(E)$.

Proof. Denote by $\Gamma$ the set of all sequences $\gamma=\left(\gamma_{i}\right)$ which can be represented in the form

$$
\gamma_{i}=\sup \left\{\alpha(p) a_{i, p}: p \in \mathbb{N}\right\}
$$

where $\alpha=\alpha(p)$ runs over the set of all non-increasing functions such that

$$
\lim _{p \rightarrow \infty} \alpha(p) a_{i, p}=0
$$

for every $i \in \mathbb{N}$ and $\alpha(p) \leq 1$. Then the collection

$$
\{\mathbf{B}(\gamma), \gamma \in \Gamma\}
$$

forms a base of $\mathcal{B}(E)$.

We check first that each set (3.2) is bounded. Indeed, if $\left(p_{i}\right) \in \Pi$ then for each $p \in \mathbb{N}$ there is $i_{0}$ such that $p \leq p_{i}$ for $i \geq i_{0}$; therefore we get that

$$
\mathbf{B}\left(\left(a_{i, p_{i}}\right)\right) \subset C U_{p} \quad \text { where } \quad C=\sup \left\{\frac{a_{i, p}}{a_{i, p_{i}}}: i<i_{0}\right\} .
$$

Thus the set $\mathbf{B}\left(a_{i, p_{i}}\right)$ is absorbed by any zero neighborhood, hence it is bounded.

Now we show that the collection (3.2) is a base of bornology $\mathcal{B}(E)$. Without loss of generality we assume that $a_{i, p}>0$ for all $(i, p) \in \mathbb{N}^{2}$. Since $E$ is Montel, each set (3.3) is precompact, hence for every $p \in \mathbb{N}$ we have

$$
\frac{\gamma_{i}}{a_{i, p}} \longrightarrow \infty \quad \text { as } \quad i \longrightarrow \infty
$$


Hence there exists a strictly increasing sequence $k(p) \uparrow \infty$ such that

$$
a_{i, p} \leq \gamma_{i}, \quad i \geq k(p)
$$

Define a sequence $\left(q_{i}\right) \in \Pi$ as follows: $q_{i}:=p$ if $k(p) \leq i<k(p+1)$ for $p \in \mathbb{N}$ and $q_{i}:=1$ if $1 \leq i \leq k(1)$. Then, by the construction,

$$
a_{i, q_{i}} \leq \gamma_{i}, \quad i \geq k(1) .
$$

Therefore for every $\gamma \in \Gamma$ there is a sequence $\left(q_{i}\right) \in \Pi$ and a constant $C>0$ such that

$$
a_{i, q_{i}} \leq C \gamma_{i}, \quad i \in \mathbb{N}
$$

which means that the collection (3.2) forms a base of bornology $\mathcal{B}(E)$.

Proof of Theorem 3.2. Due to Theorem 2.5, we can assume that there is $M \in \mathcal{B}(E)$ and a strictly increasing function $\rho \in \mathbf{N}^{\mathbf{N}}$ such that for every $p \in \mathbf{N}$ the inclusion (2.9) holds. Applying its dual form to the elements of the canonical basis $e_{i}^{\prime}$ we obtain

$$
\frac{1}{a_{i, \rho(p)}} \leq \psi(t) \frac{1}{a_{i, M}}+\frac{1}{t a_{i, p}}, \quad t>0, \quad p \in \mathbf{N},
$$

where $a_{i, M}:=\left|e_{i}\right|_{M}$. Hence we can find a map $\sigma \in \mathbf{N}^{\mathbf{N}}$ such that

$$
\frac{1}{a_{i, q}} \leq \psi(t) \frac{1}{a_{i, M}}+\frac{1}{t a_{i, \sigma(q)}}, \quad t>0, \quad q \geq \rho(1) .
$$

For an arbitrary $L \in \mathcal{B}(E)$, by Lemma 3.3, we can choose a sequence $\left(q_{i}\right) \in \Pi$ and a constant $D>0$ so that

$$
\left|x^{\prime}\right|_{L}^{*} \leq D \sup \left\{\frac{\left|\xi_{i}^{\prime}\right|}{a_{i, q_{i}}}: i \in \mathbf{N}\right\}
$$

where $x^{\prime}=\left(\xi_{i}^{\prime}\right)$. Letting $q=q_{i}$ in Eq. (3.4) we get

$$
\frac{1}{a_{i, q_{i}}} \leq \psi(t) \frac{1}{a_{i, M}}+\frac{1}{t a_{i, r_{i}}}, \quad i \in \mathbf{N}, \quad t>0,
$$

with $r_{i}=\sigma\left(q_{i}\right)$. Hence, taking into account Eq. (3.5), we obtain

$$
\left|x^{\prime}\right|_{L}^{*} \leq D \psi(t)\left|x^{\prime}\right|_{M}^{*}+\left|x^{\prime}\right|_{K}^{*}, \quad x^{\prime} \in E^{\prime}, \quad t>0
$$

with $K:=D \cdot \mathbf{B}\left(\left(a_{i, r_{i}}\right)\right) \in \mathcal{B}(E)$. So, there is $M \in \mathcal{B}(E)$ such that for any $L \in \mathcal{B}(E)$ there is $K \in \mathcal{B}(E)$ and $D>0$ such that Eq. (3.6) holds, which means that $E^{\prime} \in\left(D N_{\psi}\right)$. If the condition (2.10) holds, we can take $\psi=\varphi$ here, due to Proposition 2.8.

Since any Montel space is reflexive, the last theorem may be considered as a partial converse to Theorem 3.1. There is a natural dual relationship between the properties $s L N$ and $\overline{s Q N}(s Q N$, if $E$ is quasi-barrelled).

Proposition 3.4 For $E \in(s L N)$ it is necessary and sufficient that $E^{\prime} \in(\overline{s Q N})$.

Pr o of. Indeed, the relation (2.12) can be expressed equivalently in the form

$$
L^{\circ} \subset \Delta U_{p}^{\circ}+\delta M^{\circ},
$$

preserving all parameters and quantifiers in the definition of $s L N$. But this just means that $E^{\prime} \in(\overline{s Q N})$.

Now we deal with the duality for quasi- and asymptotical normability.

Theorem 3.5 If $E \in(p A N)$ then $E^{\prime} \in(\overline{s Q N})$ and, consequently, $E^{\prime}$ belongs to the classes $(s Q N)$ and $(Q N)$. 
This fact follows from Proposition 3.4 and the next lemma.

Lemma 3.6 If $E \in(p A N)$ then $E \in(s L N)$.

Proof. Since $E \in(p A N)$, the constant $\Delta$ in Eq. (2.11) may be chosen in the form $\Delta=C(q) D(\delta)$. Without loss of generality we can assume that $\rho(q) \geq q, q \in P$, so that $\left\{U_{r}: r \in R:=\rho(P)\right\}$ is a base of neighborhoods of 0 . Given $M \in \mathcal{B}(E)$ we choose a function $\alpha(r)$ (we assume that $\alpha(r) C(\sigma(r)) \leq 1$ and $\alpha(q) \rightarrow 0$ as $q \rightarrow \infty$ ) so that

$$
|x|_{M} \geq \sup \left\{\alpha(r)|x|_{r}: r \in R\right\} .
$$

Then, multiplying the inequality by $\alpha(\rho(q))$ and taking the supremum by $q$, we obtain

$$
|x|_{L} \leq D(\delta)|x|_{p}+\delta|x|_{M}, \quad t>0, \quad x \in E,
$$

with $L:=\left\{x \in E: \alpha(\rho(q))|x|_{q} \leq 1, r \in R\right\} \in \mathcal{B}(E)$. Thus, $E \in(s L N)$.

Remark 3.7 If $E$ in Theorem 3.5 is metrizable then $p A N$ can be substituted by $A N$.

\section{Quasi-normability and $(G)$-condition}

We denote by $\mathcal{N}$ the class of all Fréchet spaces $E$ with an unconditional basis $\left\{e_{i}\right\}$ satisfying the following conditions: there is a fundamental non-decreasing sequence of seminorms $\left\{|\cdot|_{p}\right\}$ in $E$ such that

$\mathrm{N} 1:$ there is a strictly increasing function $h: \mathbb{R}^{+} \rightarrow \mathbb{R}^{+}$such that the inequality $\left|e_{i}\right|_{q} \leq t\left|e_{i}\right|_{p}, t>0, i \in J \subset \mathbb{N}$, implies the estimate $|x|_{q} \leq C h(t)|x|_{p}$ for all $x \in E_{J}:=\overline{\operatorname{span}}\left\{e_{i}\right\}_{i \in J}$ with some constant $C=C(p, q)$, $p \leq q$

$\mathrm{N} 2$ : there is a strictly increasing function $g: \mathbb{R}^{+} \rightarrow \mathbb{R}^{+}$, converging to 0 as $t \rightarrow 0$, such that the inequality $\left|e_{i}\right|_{p} \leq t\left|e_{i}\right|_{q}, t>0, i \in J \subset \mathbb{N}$, implies the estimate $|x|_{p} \leq C g(t)|x|_{q}$ for all $x \in E_{J}:=\overline{\operatorname{span}}\left\{e_{i}\right\}_{i \in J}$ with some constant $C=C(p, q), p \leq q$.

It is worth noticing that $\mathrm{N} 1$ is related to the condition " $c \Omega$ without the restriction $k<t$ " from [6].

The next statement is a generalization of Bierstedt-Meise-Summers' result (see [4, Theorem 3.4 and Proposition 3.9]) about characterization of quasi-normable Köthe spaces in terms of the condition $(G)$, which is an improvement of the original Grothendieck's claim [12, p. 102] (see also [7]).

Theorem 4.1 A space $E \in \mathcal{N}$ is quasi-normable if and only if

(G) for each $p \in \mathbb{N}$ there is $q \in \mathbb{N}$ such that for any $\varepsilon>0$ there is $J \subset \mathbb{N}$ providing

(a) the induced topology on $E_{J}$ coincides with the p-topology, and

(b) $U_{q} \subset E_{J}+\varepsilon U_{p}$.

Proof. Let $E \in \mathcal{N},\left\{e_{i}\right\}$ be a corresponding unconditional basis in $E$, and $\left\{e_{i}^{\prime}\right\}$ be its biorthogonal system in $E^{\prime}$. Without loss of generality we can assume that the fundamental system of norms $\left\{|\cdot|_{p}\right\}_{p \in \mathbb{N}}$ satisfies the condition

$$
\left|\sum_{i \in J} e_{i}^{\prime}(x) e_{i}\right|_{p} \leq|x|_{p}
$$

for any $J \subset \mathbb{N}$.

The "if-part" is quite obvious, so we consider the "only if-part". Suppose that $E$ is quasi-normable. Then, by Meise-Vogt [18], $\left(E \in \Omega_{\varphi}\right)$ for some strictly increasing function $\varphi: \mathbb{R}^{+} \rightarrow \mathbb{R}^{+}$. Therefore, applying the condition (2.2) in a multiplicative form ([18, Theorem 7]) to the functionals $e_{i}^{\prime}$, we get that for any $p$ there is $q$ such that for every $r$ one can choose a constant $C>0$ so that

$$
\left|e_{i}^{\prime}\right|_{q}^{*} \leq C \varphi\left(\frac{\left|e_{i}^{\prime}\right|_{p}^{*}}{\left|e_{i}^{\prime}\right|_{q}^{*}}\right)\left|e_{i}^{\prime}\right|_{r}^{*}, \quad i \in \mathbb{N} .
$$


Since, due to Eq. (4.1),

$$
\left|e_{i}^{\prime}\right|_{p}^{*}=\frac{1}{\left|e_{i}\right|_{p}}
$$

the above inequality can be written as

$$
\frac{\left|e_{i}\right|_{r}}{\left|e_{i}\right|_{q}} \leq C \varphi\left(\frac{\left|e_{i}\right|_{q}}{\left|e_{i}\right|_{p}}\right), \quad i \in \mathbb{N} .
$$

For an arbitrary $\varepsilon>0$ we find $\delta>0$ such that

$$
h(\delta)<\varepsilon .
$$

Then we set $J:=\left\{i: \delta\left|e_{i}\right|_{q} \leq\left|e_{i}\right|_{p}\right\}$ and $E_{J}:=\overline{\operatorname{span}\left\{e_{i}\right\}_{i \in J}}$. From Eq. (4.2) we get that

$$
\left|e_{i}\right|_{r} \leq C \varphi\left(\frac{\left|e_{i}\right|_{q}}{\left|e_{i}\right|_{p}}\right)\left|e_{i}\right|_{q} \leq C \varphi\left(\frac{1}{\delta}\right) \delta^{-1}\left|e_{i}\right|_{p}
$$

for each $i \in J$. Therefore, taking into account the condition N1, we obtain that

$$
|x|_{r} \leq h\left(C \varphi\left(\frac{1}{\delta}\right) \delta^{-1}\right)|x|_{p}
$$

for all $x \in E_{J}$. So, the condition $(a)$ is fulfilled for the chosen subspace $E_{J}$.

On the other hand, $\left|e_{i}\right|_{p}<\delta \|\left. e_{i}\right|_{q}$ for $i \notin J$. Therefore, using the condition N2 and Eq. (4.3), we obtain that

$$
|x|_{p} \leq h(\delta)|x|_{q} \leq \varepsilon|x|_{q}
$$

holds for all $x \in E_{\mathbb{N} \backslash J}$, which means that the condition $(b)$ is true, too.

Acknowledgements The authors are grateful to J. Bonet, L. Frerick, and J. Wengenroth for their important criticism and constructive suggestions.

\section{References}

[1] A. Aytuna, Stein Manifolds $M$ for which $\mathcal{O}(M)$ is isomorphic to $\mathcal{O}\left(\Delta^{n}\right)$ as Fréchet spaces, Manuscripta Math. 62, 297-315 (1988).

[2] A. Aytuna, On Stein Manifolds $M$ for which $\mathcal{O}(M)$ is isomorphic to a power series spaces, in: Advances in the Theory of Fréchet Spaces, edited by T. Terzioğlu (Kluwer Academic Publishers, Dodrecht - Boston - London, 1988), pp. 193202.

[3] K.-D. Bierstedt, R. Meise, and W. H. Summers, A projective description of weighted inductive limits, Trans. Amer. Math. Soc. 272, 108-160 (1982).

[4] K.-D. Bierstedt, R. Meise, and W. H. Summers, Köthe sets and Köthe sequence spaces, in: Functional Analysis, Holomorphy and Approximation Theory, edited by J. A. Barroso, North-Holland Mathematics Studies Vol. 71 (NorthHolland, Amsterdam, 1982), pp. 27-91.

[5] J. Bonet, A question of Valdivia on quasi-normable Fréchet spaces, Canad. Math. Bull. 34, 301-304 (1991).

[6] J. Bonet and J. C. Diaz, The problem of topologies of Grothendieck and the class of Fréchet T-spaces, Math. Nachr. 150, 109-118 (1991).

[7] J. Bonet and J. C. Diaz, On the weak quasi-normability condition of Grothendieck, Tr. J. Math. 15, 154-164 (1991).

[8] J. Bonet and S. Dierolf, On distinguished Fréchet spaces, in: Progress in Functional Analysis, edited by K.-D. Bierstedt et al, North-Holland Mathematics Studies Vol. 170 (North-Holland, Amsterdam, 1992), pp. 201-214.

[9] E. Dubinsky and D. Vogt, Fréchet spaces with quotients failing the bounded approximation property, Studia Math. 81, 71-77 (1985).

[10] A. Goncharov and V. Zahariuta, Linear topological invariants and spaces of infinitely differentiable functions, Mat. Anal. i ego Prilozhen. (Rostov State University), 18-27 (1985).

[11] A. Grothendieck, Sur les espaces (F) et (DF), Summa Brazil Math. 3, 57-122 (1954). 
[12] A. Grothendieck, Produits Tensoriels Topologiques et Espaces Nucléaires, Memoirs of the American Mathematical Society Vol. 16 (Amer. Math. Soc., Providence, RI, 1955).

[13] H. Jarchow, Locally Convex Spaces (Teubner, Stuttgart, 1981).

[14] M. Kocatepe and V. Zahariuta, Köthe space modeled on $C^{\infty}$-spaces, Studia Math. 121, 1-14 (1996).

[15] G. Köthe, Topological Vector Spaces I (Springer, Berlin - Heidelberg - New York, 1969).

[16] G. Köthe, Topological Vector Spaces II (Springer, Berlin - Heidelberg - New York, 1979).

[17] R. Meise and D. Vogt, Introduction to Functional Analysis (Clarendon Press, Oxford, 1997).

[18] R. Meise and D. Vogt, A characterization of the quasi-normable Fréchet spaces, Math. Nachr. 122, 141-150 (1985).

[19] B. Mityagin, Approximative dimension and bases in nuclear spaces, Russian Math. Surveys 16, 59-127 (1961).

[20] B. Mityagin, Equivalence of bases in Hilbert scales, Studia Math. 37, 111-137 (1971).

[21] B. Mityagin and G. Henkin, Linear problems of complex analysis, Russian Math. Surveys 26, $99-164$ (1972).

[22] S. Önal and T. Terzioğlu, Unbounded linear operators and nuclear Köthe spaces, Arch. Math. (Basel) 11, 576-581 (1990).

[23] S. Önal and T. Terzioğlu, Concrete subspaces and quotient spaces of locally convex spaces and completing sequences, Dissertationes Math. (Rozprawy Mat.) 118, 1-36 (1992).

[24] T. Terzioğlu and D. Vogt, Some normability conditions on Fréchet spaces, Revista Matematica 2, 213-216 (1989).

[25] T. Terzioğlu and D. Vogt, On asymptotically normable Fréchet spaces, Note Mat. 11, 289-296 (1991).

[26] M. Tidten, An example of a continuum of pairwise non-isomorphic spaces of $C^{\infty}$-spaces, Studia Math. 78, 267-274 (1984).

[27] M. Valdivia, On quasi-normable echelon spaces, Proc. Edinb. Math. Soc. (2) 24, 73-80 (1981).

[28] D. Vogt, Eine Charakterisierung der Potenzreihenräume von endlichen Typ und ihre Folgerungen, Manuscripta Math. 37, 269-301 (1982).

[29] D. Vogt, Some results on continuous linear maps between Fréchet spaces, in: Functional Analysis: Surveys and Recent Results II, edited by K.-D. Bierstedt and B. Fuchssteiner, Noth-Holland Mathematics Studies Vol. 90 (North-Holland, Amsterdam, 1984), pp. 349-381.

[30] D. Vogt, Remarks on a paper of S. Önal and T. Terzioğlu, Tr. J. Math. 15, 200-204 (1991).

[31] D. Vogt and M. J. Wagner, Charakterisierung der Quotientenräume von $s$ und eine Vermutung von Martineau, Studia Math. 67, 225-240 (1980).

[32] V. Zahariuta, Quasi-equivalence of bases in finite centers of Hilbert spaces, Soviet. Math. Dokl. 9, 681-684 (1968).

[33] V. Zahariuta, Extremal plurisubharmonic functions, Hilbert scales, and isomorphisms of spaces of analytic functions of several variables, I, Teor. Funktsiŭ Funktsional. Anal. i Prilozhen. 19, 133-157 (1974) (in Russian).

[34] V. Zahariuta, Extremal plurisubharmonic functions, Hilbert scales, and isomorphisms of spaces of analytic functions of several variables, II, Teor. Funktsiŭ Funktsional. Anal. i Prilozhen. 21, 65-83 (1974) (in Russian).

[35] V. Zahariuta, On isomorphic classification of $F$-spaces, in: Linear and Complex Analysis Problem Book, 199 Research Problems, Lecture Notes in Mathematics Vol. 1043 (Springer, Berlin-Heidelberg-New York, 1984), pp. 34-37.

[36] V. Zahariuta, Spaces of analytic functions and complex potential theory, in: Linear Topological Spaces and Complex Analysis, I, edited by A. Aytuna (METU-TÜBİTAK, Ankara, 1994), pp. 74-146. 